\title{
Hypothesis
}

\section{Identifying Candidate Genes for Blood Pressure Quantitative Trait Loci Using Differential Gene Expression and a Panel of Congenic Strains}

\author{
George T. Cicila and Soon Jin Lee
}

\begin{abstract}
The most difficult step in dissecting the molecular basis of a quantitative trait is proceeding from chromosomal locations associated with this trait (i.e., quantitative trait locus, QTL) to determining what gene(s) in the QTL region is causative. Using standard positional cloning methodology to identify candidate genes for a particular QTL has three drawbacks: 1) it is labor intensive; 2) defining variants in genes causing quantitative variation from sequence information alone is difficult or impossible; and 3 ) many (or most) genes in a particular chromosomal interval will not be relevant for a specific disease/trait because they are not expressed in critical candidate organs. Instead of positional cloning, we propose using a panel of congenic strains, where each carries an allele for a different QTL on a similar genetic background, in conjunction with identification of differentially-expressed genes in target organs of inbred strains of contrasting phenotype. This will identify genes having altered expression in organs critical to regulating blood pressure and the development of hypertension. Radiation hybrid mapping of such genes will result in a transcription map of differentially-expressed genes in a target organ of a rat model of genetic hypertension. This approach could rapidly identify genes mapping to genomic regions near QTL, which will be strong candidates to explain, in part, the observed strain differences in blood pressure. This novel approach, which uses a panel of congenic strains to facilitate the mapping and subsequent identification of differentially-expressed and QTL-associated genes, should be applicable to any genetic model for identifying candidate genes located near QTL, given the availability of a panel of congenic strains. (Hypertens Res 1998; 21: 289-296)
\end{abstract}

Key Words: radiation hybrid mapping, hypertension, congenic strains, differential mRNA expression, Dahl rat

Human essential hypertension is a complex, multifactorial disorder resulting from the interplay of multiple environmental and genetic factors. Several animal models of genetic hypertension have been developed to unravel the genetic determinants of blood pressure regulation. Identifying genes that predispose animals to high blood pressure will lead to elucidation of factors influencing blood pressure and related quantitative traits in humans.

Genetic Models of Blood Pressure Salt-Sensitivity and Salt-Resistance

The inbred Dahl salt sensitive (S) rat is the most widely studied genetic model of salt-sensitive hypertension. In this strain, supplemental dietary $\mathrm{NaCl}$ increases blood pressure, whereas in the Dahl saltresistant (R) strain, supplemental dietary $\mathrm{NaCl}$ has little or no effect $(1,2)$. Dahl $\mathrm{S}$ rats are also genetically-hypertensive, and high blood pressures develop over time, even when given low or normal levels of dietary $\mathrm{NaCl}$. Thus, $\mathrm{S}$ rats can be used to model the influence of genetic factors, as well as genetic-environmental interactions, affecting blood pressure. Segregating populations derived from these inbred strains has been used to screen for genes leading to heritable differences in blood pressure as well as genes underlying the gene $\times$ environment interactions responsible for blood pressure salt-sensitivity and salt-resistance.

\section{Congenic Strains and Blood Pressure Quantitative Trait Locus (QTL) Analysis \\ Imprecision of localization is the major difficulty in using interval mapping to screen segregating popu- lations for QTL. Theoretical studies suggest that QTL can be mapped with confidence intervals of only about 10 to $35 \mathrm{cM}(3,4)$. Congenic strains con- structed by introgressing a small chromosomal re- gion from a donor strain that contains a QTL into a recipient strain offer potentially much more precise}

From the Department of Physiology and Molecular Medicine, Medical College of Ohio, Ohio, USA.

This work was supported by grants to G.T. Cicila from the National Institutes of Health (HL52698) and the Ohio/West Virginia Affiliate of the American Heart Association (NW-97-04-S).

Address for Reprints: George T. Cicila, Ph.D., Medical College of Ohio, Block Health Science Building, Department of Physiology and Molecular Medicine, 3035 Arlington Avenue, Toledo, Ohio 43614, USA.

Received May 8, 1998; accepted in revised form August 3, 1998. 
QTL localization (5), by establishing definitive limits for a region containing a QTL. These limits can be further narrowed by selecting congenic substrains retaining the QTL with progressively smaller amounts of donor chromosome (5).

This strategy has proved effective for confirming blood pressure QTL locations in rat models. We recently used congenic strains created by introgressing regions of R-rat chromosome containing putative chromosome 7 and 3 blood pressure QTL $(6,7)$ into a background of S-rat-derived alleles to confirm the location of these blood pressure QTL $(8,9)$. Others in our department have used congenic strains to confirm blood pressure QTL located on rat chromosomes $1(10), 2(11), 5(10), 9(12), 10$ $(10,13)$, and $13(14)$. A number of congenic strains have also been developed by other groups $(15-19)$ in this and other rat models of genetic hypertension. Additional uses of such panels of congenic strains will be discussed in detail later.

\section{Identification of Candidate Genes for Blood Pressure $Q T L$}

The strongest impediment to understanding the molecular basis of hypertension (and indeed, of all quantitative traits) is proceeding from chromosomal locations associated with a trait (i.e., locating QTL) to determining what gene(s) in that chromosomal region associated with the QTL is causative of the trait. Altered expression of a gene(s) (whether in protein activity, mRNA expression level, or both) will likely underlie heritable quantitative traits. Using positional cloning techniques to identify candidate genes for a particular QTL has the following drawbacks: 1) it is labor intensive; 2) defining genes causing quantitative variation from sequence information alone using computer programs is difficult or impossible; and 3) many (or most) genes in a particular chromosomal interval will not be relevant for studying a specific disease/trait because they are not expressed in critical target organs.

A number of systematic methods to screen for genes having altered levels of mRNA expression in a particular organ or cell type have been recently developed, including differential display of mRNA (20), RNA fingerprinting (21), serial analysis of gene expression (SAGE) (22), representational difference analysis (RDA) (23), and arrays of immobilized cDNA clones (24). While differential display of mRNA has been used to identify genes with tissue-specific expression for subsequent genomic mapping $(25,26)$, no one has used mRNA expression patterns in congenic strains to facilitate the identification and mapping of differentially-expressed and QTL-associated genes.

Markers derived from differentially-expressed genes allow us to directly examine genes expressed in organs thought to be involved in the regulation of blood pressure. This avoids the time and effort of examining all genes located in chromosomal intervals (25) containing blood pressure QTL introgressed into congenic strains, because many (or most) genes in a given chromosomal interval will not be 1) expressed in organs critical for the phenotypic trait or
2) differentially-expressed in those organs.

We propose to identify a subset of genes that are differentially-expressed in a critical target organ, which will be selected based on their mRNA expression pattern (described below) in a panel of congenic strains, where each strain harbors a different blood pressure QTL. Markers will be developed for this subset of genes and used to determine their genomic location. This procedure will allow direct screening for gene(s) whose 1) altered mRNA expression in a critical organ and 2) genomic location make them strong candidate genes for a particular congenic strain's blood pressure QTL. This would result in a novel resource, a transcription map of genes that are differentially-expressed between strains having contrasting phenotypes in a target organ of a genetic model of hypertension.

\section{Hypothesis}

We hypothesize that gene(s) underlying the observed blood pressure QTL may be differentiallyexpressed in target organs/tissues between strains having contrasting phenotypes. If this is so, such genes should also be differentially-regulated in congenic strains carrying different blood pressure QTL. Genes differentially expressed in only a single congenic strain as compared with recipient rats may be involved in a pathway of blood pressure regulation limited to a particular congenic strain. Gene(s) responsible for a particular QTL's effect should show a congenic strain-specific differential pattern of expression in a candidate organ(s) and should map to the chromosomal interval carried by that particular congenic strain.

\section{Approach}

\section{Identifying and Screening Differentially Expressed Genes}

We first have to determine which organs or tissues are most likely to identify the key, differentially-expressed genes that are causative for our quantitative trait of interest. Logical places to search for altered expression of "causative" genes are organs that are critical in the regulation of blood pressure and the development of hypertension, which we will define as candidate organs. Kidney, brain, heart, and aorta are candidate organs that quickly come to mind: the kidney for its key role in maintaining salt and water balance; the brain for its central role in the neural and hormonal control of blood pressure; and the heart and aorta for factors that can produce remodeling in response to the development of hypertension. Other organs/tissues, such as adrenal glands or ganglia of the sympathetic nervous system, clearly would also be suitable for such analysis.

We will then identify genes that are differentiallyexpressed, using one of the above-mentioned methodologies, between the hypertensive strain and an appropriate normotensive strain (or strains) in the chosen candidate organ. For complex organs such as the kidney and the brain, we can legitimately expect dozens of genes, if not hundreds or more, 
Table 1. Selected Characteristics of Congenic Strains Containing Blood Pressure QTL from Dahl Rats

\begin{tabular}{|c|c|c|c|c|c|c|}
\hline Chromosome & $N$ & $\begin{array}{l}\text { Donor } \\
\text { Strain }^{\mathrm{a}}\end{array}$ & $\begin{array}{l}\text { Recipient } \\
\text { Strain }^{\mathrm{a}}\end{array}$ & $\begin{array}{c}\text { Congenic Blood Pressure } \\
\text { Effect }(\mathrm{mmHg})^{\mathrm{b}}\end{array}$ & $\begin{array}{c}p \\
(t \text { test })\end{array}$ & Ref. \\
\hline 1 & S.LEW(1) & LEW & $\mathrm{S}$ & 26 & $<0.0001$ & $(10)$ \\
\hline 2 & S.WKY(2) & WKY & $\mathrm{S}$ & 32 & $<0.0001$ & (11) \\
\hline 3 & S.R-Edn3 & $\mathrm{R}$ & S & 21 & 0.0001 & (9) \\
\hline 5 & S.LEW(5) & LEW & $S$ & 26 & $<0.0001$ & (10) \\
\hline 7 & S.R-Cyp11b & $\mathrm{R}$ & S & $63^{c}$ & $<0.0001^{\mathrm{c}}$ & $(8)$ \\
\hline 9 & S.R-Inha & $\mathrm{R}$ & $S$ & 19 & $<0.0001$ & (12) \\
\hline \multirow[t]{2}{*}{10} & S.M(10b) & MNS & $\mathrm{S}$ & 39 & $<0.0001$ & (13) \\
\hline & S.LEW(10) & LEW & $\mathrm{S}$ & 42 & $<0.0001$ & $(10)$ \\
\hline 13 & S.R-Ren & $\mathrm{R}$ & S & 25 & 0.002 & (14) \\
\hline 2 and 10 & S.WKY(2)M(10b) & WKY/MNS & $\mathrm{S}$ & 47 & $<0.0001$ & (27) \\
\hline
\end{tabular}

anbred rat strain names are abbreviated as follows: LEW, Lewis; WKY, Wistar-Kyoto; R, Dahl salt-resistant; MNS, Milan normotensive strain; S, Dahl salt-sensitive. ${ }^{b}$ Blood pressure was measured after $37-\mathrm{d}$ old male rats were fed a $2 \%$ $\mathrm{NaCl}$ diet for $25 \mathrm{~d}$. Blood pressure effect is the decrease in systolic blood pressure of the congenic strain compared to $\mathrm{S}$ rats, tested concomitantly. ${ }^{\text {T }}$ These rats were fed a $4 \% \mathrm{NaCl}$ diet.

to show significantly altered mRNA expression levels in the hypertensive as compared with the control strains. The bigger question becomes how can we select among the differentially-expressed genes to identify those that are the strongest candidate genes, i.e., those most likely to cause blood pressure QTL.

We propose using the panels of congenic strains, which are now being developed to confirm and localize blood pressure QTL (see above), to screen genes that are differentially-expressed in candidate organs, in order to identify those that are the strongest candidate genes for the quantitative trait of interest. A differentially-expressed gene causative of a particular blood pressure QTL would be expected to show a similar altered mRNA expression in a congenic strain that has this QTL introgressed, but not in most other congenic strains, which will have other QTL introgressed. The expression patterns of differentially-expressed genes would then be examined using Northern filter hybridization, RNase protection, or reverse transcriptase-polymerase chain reaction with RNA samples from all congenic strains studied.

Because differential expression must be confirmed owing to the possibility of false positives occurring, inclusion of several more samples requires little additional expense and effort and avoids performing the differential display technique using samples from the entire panel of congenic strains. Clearly, the approach outlined above assumes that mRNA expression of gene(s) responsible for a QTL will be conserved between the parental, normotensive strain and the congenic strain into which that gene(s) is introgressed, and that this differentiallyexpressed gene contributes to the observed differences in phenotype between the congenic rat and the parental, hypertensive strain.

\section{Patterns of Expression in the Panel of Congenic Strains}

This new paradigm will be examined in the context of the inbred Dahl rat model of genetic hypertension. We will seek genes with altered mRNA expression by comparing mRNA expression levels between congenic rat strains, each carrying a different low blood pressure QTL allele (e.g., Table 1), with the parental hypertensive strain (in the example, the Dahl S rat). Each congenic strain would be derived by introgressing a different region of a normotensive rat chromosome containing a low blood pressure QTL allele into the hypertensive S strain. This will result in a panel of congenic strains that have similar genetic backgrounds. Such a congenic strain panel has been developed, and Table 1 summarizes some of its characteristics. While congenic strains could also be derived by introgressing portions of donor chromosome containing a high blood pressure QTL allele from a hypertensive strain into a normotensive strain, the key for this sort of comparison is to have highly similar genetic backgrounds in all congenic strains in the panel. Figure 1 describes a number of possible differential mRNA expression patterns in the panel of congenic strains that may be used to identify candidate genes for specific blood pressure QTL. For this example, patterns of mRNA expression will be discussed in terms of a congenic panel bred by introgressing portions of a normotensive rat strain $(e . g ., \mathrm{R})$ chromosome into the $\mathrm{S}$ strain.

Expression of a gene(s) responsible for a particular QTL should be altered in a candidate organ(s) of a congenic strain carrying the normotensive ( $R$ in our example) rat chromosomal region containing that gene. Other congenic strains should carry the S-rat allele for that gene, whose expression in the target organ(s) would not be expected to be altered as compared with the $\mathrm{S}$ rat (pattern $\mathrm{I}$ ).

A type II pattern of expression, where several, but not all, congenic strains and the contrasting normotensive strain show altered mRNA expression as compared with $\mathrm{S}$ rats will be interpreted as stemming from a number of QTL carried by this subset of congenic strains that affect a common pathway 


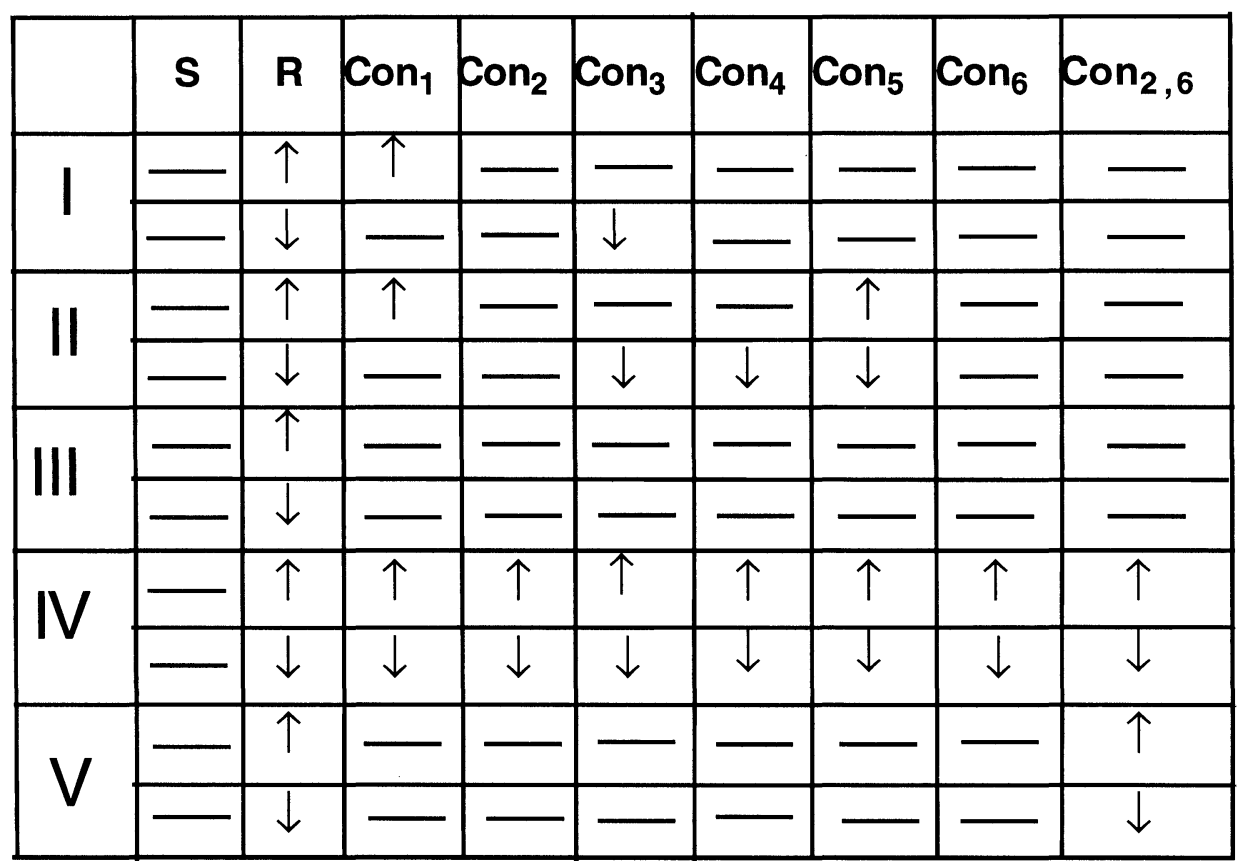

Fig. 1. Interpreting patterns of differential gene expression in a panel of congenic strains. This figure presents a hypothetical scheme for interpreting Northern hybridization results for filters containing $R N A$ from $S, R$, and a number of congenic rat strains designated $\mathrm{Con}_{1}$, Con, , etc., that were probed with a putative differentially-expressed cDNA. In this figure, congenic strains were assumed to be constructed by introgressing a portion of $R$-rat chromosome into the $S$ strain, with each congenic strain carrying a low blood pressure allele for a different blood pressure QTL. Con 2,6 represents a "double" congenic strain, where QTL found in congenic strains $\mathrm{Con}_{2}$ and Con 6 were both introgressed into the $S$ strain. RNA expression levels are all compared with $S$-rat and are designated by the following symbols: $(-)$ similar to $S$-rat expression levels, ( $\uparrow$ ) greatly increased relative to $S$-rat levels, and ( $\downarrow$ ) greatly reduced relative to $S$-rat levels. Roman numerals refer to the different patterns of $m R N A$ expression described in the text. Note that a particular pattern of expression is not limited to either upregulated or down-regulated expression of a gene in $R$ or congenic strains, or both, as compared with the $S$ strain.

leading to altered blood pressure. Altering this pathway, in turn, leads to differences in mRNA expression levels for this particular gene in this subset of the congenic panel. Another possibility is that the differentially-expressed gene is located in the chromosomal region introgressed into one of the congenic strains, but that the gene is part of a common pathway that is affected in several of the other congenic strains.

Another expected pattern is where similar levels of mRNA expression are shared by all congenic strains and the $\mathrm{S}$ rat, differing from that found in the contrasting normotensive stain (pattern III). These expression patterns are expected to be common because for most loci every congenic strain in the panel will carry the S-rat-derived allele. Assuming that each congenic strain in the panel described in Table 1 carries an introgressed region of $30 \mathrm{cM}$ out of a total genome of $1,700 \mathrm{cM}$, all congenic strains in the panel would carry the parental S-rat allele for $87 \%$ of the loci $[(1,700-30) / 1,700]^{8}$.

Genes with expression patterns I and II represent the best candidates for mapping, with those having pattern I given priority. Following this logic, a gene(s) responsible for a particular QTL will map within the chromosomal region introgressed from the normotensive rat into the congenic strain. Dif- ferentially expressed genes that map into these introgressed regions will be strong candidates to account for the observed quantitative effects "trapped" in the congenic strain. Strong candidate genes would be further tested by breeding congenic substrains carrying the donor strain allele for the candidate gene (and the QTL) with a minimal amount of flanking donor chromosomal material because of the possibility that a given QTL could stem from multiple genes.

Other, more complex patterns of expression are also possible, which will be more difficult to interpret, e.g., where all (or most) congenic strains and the normotensive strain ( $\mathrm{R}$ in this case) show similar expression levels, which differ from $\mathrm{S}$ rats. Because all of our congenic strains have lower blood pressures than $\mathrm{S}$ rats, this expression pattern (expression pattern IV, Fig. 1) is likely to reflect secondary effects stemming from these lower blood pressures, and genes showing these patterns of expression would not be studied further.

We can also consider the case of a "double" congenic strain, such as S.WKY(2)M(10b) (27), which carries two QTL (expression pattern V, Fig. 1). If a "double" congenic strain shows differential gene expression as compared with the two congenic strains from which it was bred, these patterns of altered 
RNA expression could arise from epistatic interactions between two QTL-containing regions introgressed into this "double" congenic strain, where neither QTL-containing region alone would alter this gene's expression. Such genes would clearly be worth mapping and examining in detail. Such altered expression of a gene in a "double" congenic rat could stem from the effects of one introgressed "low" blood pressure QTL allele (e.g., from the $\mathrm{Con}_{2}$ strain) to alter the expression of another "low" blood pressure QTL allele, introgressed from the other congenic strain $\left(\mathrm{Con}_{6}\right.$ in this example). In this example the differentially-expressed gene would map to the QTL-containing chromosomal region introgressed into $\mathrm{Con}_{6}$. Alternatively, epistatic interactions between genes present in both introgressed intervals (e.g., those from $\mathrm{Con}_{2}$ and from $\mathrm{Con}_{6}$ ) could result in the altered expression of a third gene, which could map outside of the QTL containing chromosomal regions introgressed into the "double" congenic strain.

Comparisons of gene expression patterns discussed above will be most useful if the panel of the congenic rats used essentially has the same genetic background to facilitate comparisons and testing of interactions using "double" congenic rats. Using congenic rats with QTL-containing regions introgressed into different genetic backgrounds, even if from similar hypertensive strains, will complicate interpretation of the gene expression patterns in a panel, because differential expression of genes may be due to the effects of different genes being present in the respective recipient strains (i.e., due to variable genetic background differences), rather than from the effects of the QTL-containing regions introgressed. Similarly, using a panel of congenic rats where QTL-containing regions from different donor strains were introgressed into the same recipient strain, as is the case in Table 1 , would not be expected to complicate interpretation to the same extent, because the genetic backgrounds of all congenic strains in the panel should be very similar. Nevertheless, in our preliminary experiments using the differential display of mRNA technique with the strains described in Table 1 we observed genes showing four of the expression patterns described above (unpublished observations). We did not observe the type IV expression pattern, perhaps because of the age of the rats ( 1 mo old) used in the above experiments, at which time large differences in blood pressure between the inbred and congenic strains would not be expected.

\section{Mapping Differentially-Expressed Genes}

Differentially-expressed genes showing altered RNA expression in only a single congenic strain or a limited subset of the congenic strain panel, will be selected for mapping. This mapping can be done physically, using a radiation hybrid cell panel (28) or genetically, using segregating rat populations bred from crosses of recipient rats with contrasting rat strains. Radiation hybrid mapping is particularly suitable for mapping differentially-expressed genes because it does not require polymorphic markers. Polymorphic markers, such as microsatellites, would be developed, however, for differentially expressed genes mapping to QTL-containing chromosomal regions, so that these candidate genes could be placed onto genetic maps.

A differentially-expressed gene that maps to a chromosomal region introgressed into a congenic strain will be a strong candidate for the QTL present in that particular strain. Thus, knowledge of the chromosomal location for a differentially-expressed gene, in conjunction with its expression pattern of target organ in the congenic strain panel, will be useful in identifying genes that are the best candidates for the blood pressure QTL. This new paradigm should identify new candidate genes for confirmed blood pressure QTL in rat models of genetic hypertension. The transcription map of genes differentially expressed in target organs resulting from this approach will be useful for studying the effects of physiological challenges, such as a high salt diet, on blood pressure (see below).

\section{Additional Applications of this Approach}

Because blood pressure is a complex phenotype, stemming from the cumulative effects of multiple genes, environmental effects, and gene-environment interactions, the paradigm described here could be extended to examining genes showing differential expression in target organs in response to alterations in diet (e.g., changing the dietary intake of $\mathrm{NaCl})$ or to pharmacological interventions that modulate blood pressure. This should be seen as a way of further characterizing known blood pressure QTLs, because this approach would only detect candidate genes present in chromosomal regions already known to contain blood pressure QTLs (and have already been introgressed into congenic strains). Clearly, identifying all QTLs associated with a particular environmental intervention (e.g., an elevated dietary $\mathrm{NaCl}$ intake or drug treatment) requires interval mapping of one or more segregating populations, derived from inbred strains having contrasting phenotypes, subjected to that environmental intervention. Such genomic scan approaches will require more animals (at least 150 to 300 rats/ population) for each environmental intervention to be tested, as compared with testing a panel of congenic rats.

Previous work should show that the environmental intervention resulted in significant strain-differences in blood pressure in the genetic model of interest, before trying such an extension of our paradigm. Genes showing strain-differences in expression in response to an environmental intervention (e.g., a high dietary $\mathrm{NaCl}$ intake) would have their RNA expression further examined in a panel of congenic strains, divided into groups either treated or not treated with the environmental intervention. As described above, genes showing altered RNA expression in response to an environmental challenge in only a single congenic strain or a limited subset of the congenic strain panel would be selected for mapping. Such genes, if they map to 
QTL-containing regions, would be good candidates for genes involved in gene-environment interactions.

Similarly, congenic strain panels could also be tested for differences in intermediate phenotypes/ physiological traits (e.g., glomelular filtration rate or fractional excretion of $\mathrm{Na}^{+}$for the kidney) that previous studies indicate are relevant to blood pressure regulation in the chosen model system and in which strain-differences are known to exist. The resulting patterns for physiclogical measurements in the congenic panel could be analyzed as described above. Transcription maps of differentially-expressed genes in target organs, in conjunction with testing of the congenic strain panel for key physiological measures, may promote understanding of the progression of pathologic changes occurring during hypertension and of possible blood pressure regulatory mechanisms. Testing of physiological variables representing key intermediate phenotypes may be very costly or time consuming to set up.

Identifying genes differentially-expressed in a subset of the congenic strains may be useful in identifying common pathways affecting the regulation of blood pressure. Differential-gene expression observed in a subset of congenic strains represents a situation where different sets of genes, which are introgressed into each congenic strain, affect the expression of a single gene. The differentially-expressed gene can be located in, at most, one introgressed QTL-containing region, unless it is a member of a multi-gene family with a high degree of sequence similarity. Also, identification of several genes that are differentially-expressed in the same subset of the congenic panel may suggest the existence of a novel intermediate phenotype to explore.

This paradigm need not be limited to examining differences in mRNA expression levels using purified RNA samples isolated from the target organs of congenic rats. The regional expression of genes identified by differential display of mRNA could be studied in tissue sections of target organs prepared from the different congenic strains using in situ hybridization or immunohistochemical approaches. Thus, this approach could be used to examine the panel of congenic strains for localized or tissue-wide differences in mRNA or protein expression. Examination of tissue sections from the target organ isolated from the congenic strains in this panel could be used to identify congenic strain-specific differences in the size or appearance of histologic structures.

\section{Possible Pitfalls of this Approach}

This approach is not without shortcomings. The biggest is its inability to detect mutations that might lead to phenotypic differences without any detectable alteration of mRNA expression levels. Such mutations would include point mutations altering the amino acid sequence, as well as mutations affecting the post-transcriptional modification of RNA, such as alternative splicing, or the post-translational modification of proteins. As such, the paradigm described here is incapable of identifying all of the possible mutations that could potentially result in a quantitative trait locus. Indeed, point mutations in genes have been linked to altered blood pressure both in animal models of hypertension $(6,8)$ and in Mendelian forms of human hypertension (e.g., 29, reviewed in 30). Establishing a mass-screening approach to identify such point mutations, particularly for uncharacterized genes, seems at best to be problematical.

However, mutations that affect the activity of a protein often result in altered gene expression to compensate for the altered activity. For a disorder such as essential hypertension, where the cause is not due to obvious lesions in a particular gene or organ, genetic alterations would be expected to be subtle, such as up-regulated or down-regulated expression of an active gene, or point mutations that alter, but do not abolish, a protein's activity. It is therefore reasonable to expect many, if not most, of the genes underlying blood pressure QTL to be differentially expressed in key organs, such as the kidney.

Another potential weakness with this paradigm is that for complex organs such as the brain and kidney it is possible that a gene could be up-regulated in some cell types, but down-regulated elsewhere in the same organ, or vice versa. Certainly, while particularly interesting cell types or portions of organs can be addressed using cell culture or micro-dissection techniques, neither approach seems particularly well suited for a screening approach, as is outlined here.

\section{Blood Pressure Candidate Genes}

Examples of candidate genes for blood pressure QTL showing modulated gene expression include the $S_{A}$ gene, first identified by differential screening of a cDNA library (31), and steroid $11 \beta$-hydroxylase (Cyp11b1), first identified on the basis of enzymatic activity differences $(32,33)$. Both genes cosegregated with blood pressure in segregating rat populations $(6,32,34-37)$ and were confirmed by introgression into congenic strains as possible candidate genes for blood pressure $(8,10,19)$.

Little is known about the function of the $\mathrm{S}_{\mathrm{A}}$ gene-product under either physiological or pathophysiological conditions. Any effects of the $\mathrm{S}_{\mathrm{A}}$ gene on blood pressure are probably due to differential gene expression, because 1) no strain differences in the deduced amino acid sequence of this gene have been observed and 2) this gene cosegregates with renal $\mathrm{S}_{\mathrm{A}}$ mRNA expression levels in an $\mathrm{F}_{2}(\mathrm{SHR} \times$ WKY) population (36).

On the other hand, much is known about the function of Cyp11b1, an adrenal steroidogenic enzyme of the cytochrome P450 super-family. In rats, this enzyme catalyzes the adrenal conversion of deoxycorticosterone into primarily corticosterone and 18-hydroxy-11-deoxycorticosterone (18-OHDOC), with the mineralocorticoid 18-OH-DOC long known to have hypertensinogenic properties (38). Coding sequence mutations in Cyp11b1 $(6,39)$ cause the adrenal mitochondria of Dahl $\mathrm{R}$ rats to convert a lower proportion of exogenous DOC into 
18-OH-DOC, as compared with adrenal mitochondria from most other rat strains, including $\mathrm{S}$ rats, in vitro, and this altered enzymatic activity cosegregated with blood pressure in a backcross population of $\mathrm{S}$ and $\mathrm{R}$ rats $(6,32)$. Adrenal Cyp11b1 mRNA expression is also up-regulated in $\mathrm{S}$ rats as compared with $\mathrm{R}$ rats (Cicila and $\mathrm{Ng}$, unpub.), most likely because of lower production of corticosterone/mg of adrenal cortex, and compensatory ACTH release causing adrenal hyperplasia.

\section{Final Thoughts}

In conclusion, we describe how identification of differentially-expressed genes in target organs, in conjunction with a panel of congenic strains, may speed up the process of finding candidate genes involved in blood pressure regulation and perhaps suggest additional mechanisms for the genesis and progression of hypertension. The differentially-expressed genes identified by this approach are likely to stem from "subtle mutations," altering but not abolishing the gene(s)' expression level or activity (or both). This approach of analyzing patterns of expression in a panel of congenic strains should be widely applicable, including examination of differences in protein expression, physiological variables, or even histologic structures in tissue sections. Transcriptional maps of genes differentially-expressed in target organs in response to nutritional (or other) modulation may be useful in identifying genes underlying heritable differences stemming from gene-environment interactions (e.g., blood pressure salt-sensitivity or salt-resistance).

\section{Acknowledgements}

We thank Drs. J.P. Rapp, H. Dene, and S.L. Britton for critical reading and discussion of the manuscript.

\section{References}

1. Dahl LK, Heine M, Tassinari L: Effects of chronic excess salt ingestion: evidence that genetic factors play an important role in the susceptibility to experimental hypertension. J Exp Med 1962; 115: 11731190.

2. Rapp JP, Dene H: Development and characteristics of inbred strains of Dahl salt-sensitive and salt-resistant rats. Hypertension 1985; 7: 340-349.

3. Darvasi A, Weinreb A, Minke V, Weller JI, Soller $\mathrm{M}$ : Detecting marker-QTL linkage and estimating QTL gene effect and map location using a saturated genetic map. Genetics 1993; 134: 943-951.

4. Hyne V, Kearsey MJ, Pike DJ, Snape JW: QTL analysis: unreliability and bias in estimation procedures. Mol Breeding 1995; 1: 273-282.

5. Rapp JP, Deng AY: Detection and positional cloning of blood pressure quantitative trait loci: is it possible? Identifying the genes for genetic hypertension. Hypertension 1995; 25: 1121-1128.

6. Cicila GT, Rapp JP, Wang J-M, St. Lezin E, Ng SC, Kurtz TW: Linkage of $11 \beta$-hydroxylase mutations with altered steroid biosynthesis and blood pressure in the Dahl rat. Nat Genet 1993; 3: 346-353.

7. Cicila GT, Rapp JP, Bloch KD, et al: Cosegregation of the endothelin-3 locus with blood pressure and rel- ative heart weight in inbred Dahl rats. $J$ Hypertens 1994; 12: 643-650.

8. Cicila GT, Dukhanina OI, Kurtz TW, et al: Blood pressure and survival of a chromosome 7 congenic strain derived from Dahl rats. Mamm Genome 1997; 8: 896-902.

9. Cicila GT, Choi C, Dene H, Lee SJ, Rapp JP: Two blood pressure/cardiac mass quantitative trait loci are present on chromosome 3 of Dahl salt-sensitive and salt-resistant rats. Mamm Genome 1998 (in press).

10. Garrett MP, Dene H, Walder R, et al: Genomic scan and congenic strains for blood pressure QTL using Dahl salt-sensitive rats. Genome Res 1998 (in press).

11. Deng AY, Dene H, Rapp JP: Congenic strains for blood pressure quantitative locus on rat chromosome 2. Hypertension 1997; 30 (Pt1): 199-202.

12. Rapp JP, Garrett MR, Dene H, Meng H, Hoebee B, Lathrop GM: Linkage analysis and construction of a congenic strain for a blood pressure QTL on rat chromosome 9. Genomics 1998 (in press).

13. Dukhanina OI, Dene $\mathrm{H}$, Deng AY, Choi CR, Hoebee B, Rapp JP: Linkage map and congenic strains to localize blood pressure QTL on rat chromosome 10. Mamm Genome 1997; 8: 229-235.

14. Zhang Q-Y, Dene H, Deng AY, Garrett MR, Jacob HJ, Rapp JP: Interval mapping and congenic strains for a blood pressure QTL on rat chromosome 13 . Mamm Genome 1997; 8: 636-641.

15. Pravenec M, Klìr P, Křen V, Zicha J, Kunes J: An analysis of spontaneous hypertension in spontaneously hypertensive rats by means of new recombinant inbred strains. J Hypertens 1989; 7: 217-222.

16. St Lezin EM, Pravenec M, Wong AL, et al: Effects of renin gene transfer on blood pressure and renin gene expression in a congenic strain of Dahl salt-resistant rats. J Clin Invest 1996; 97: 522-527.

17. Jiang J, Stec DE, Drummond $\mathrm{H}$, et al: Transfer of a salt-resistant renin allele raises blood pressure in Dahl salt-sensitive rats. Hypertension 1997; 29: 619627.

18. Kren V, Pravenec M, Lu S, et al: Genetic isolation of a region of chromosome 8 that exerts major effects on blood pressure and cardiac mass in the spontaneously hypertensive rat. J Clin Invest 1997; 99: 577-581.

19. St Lezin E, Liu W, Wang J-M, et al: Genetic isolation of a chromosome 1 region affecting blood pressure in the spontaneously hypertensive rat. Hypertension 1997; 30: 854-859.

20. Liang P, Pardee AB: Differential display of eukaryotic messenger RNA by means of the polymerase chain reaction. Science 1992; 257: 967-971.

21. Welsh J, Chada K, Dalai SS, Cheng R, Ralph D, McClelland M: Arbitrary primed PCR fingerprinting of RNA. Nucleic Acids Res 1992; 20: 4965-4970.

22. Velculescu VE, Zhang L, Vogelstein B, Kinzler KW: Serial analysis of gene expression. Science 1995; 270: 484-487.

23. Hubank M, Schatz DG: Identifying differences in mRNA expression by representational difference analysis of cDNA. Nucleic Acids Res 1994; 22: 56405648.

24. Schena M, Shalon D, Davis RW, Brown PO: Quantitative monitoring of gene expression patterns with a complementary DNA microarray. Science 1995; 270: 467-470.

25. McClelland M, Chada K, Welsh J, Ralph D: Arbitrary primed PCR fingerprinting of RNA applied to mapping differentially expressed genes in DNA Fingerprinting in Pena SDJ, Chakraborty R, Epplen JT, Jeffreys AJ (ed): State of the Science, Basel, 
Birkhäuser Verlag, 1993, pp103-115.

26. Ferrer J, Wasson J, Schoor KD, Mueckler M, DonisKeller H, Permutt MA: Mapping novel pancreatic islet genes to human chromosomes. Diabetes 1997; 46: 389-392.

27. Rapp JP, Garrett MR, Deng AY: Construction of a double congenic strain to prove an epistatic interaction on blood pressure. J Clin Invest 1998; 101: 15911595.

28. Cox DR, Burmeister M, Price ER, Kim S, Myers RM: Radiation hybrid mapping: a somatic cell genetic method for constructing high-resolution maps of mammalian chromosomes. Science 1990; 250: 245250.

29. Lifton RP, Dluhy RG, Powers M, et al: Hereditary hypertension caused by chimaeric gene duplications and ectopic expression of aldosterone synthase. Nat Genet 1992; 2: 66-74.

30. Lifton RP: Genetic determinants of human hypertension. Proc Natl Acad Sci USA 1995; 92: 8545-8551.

31. Iwai $\mathrm{N}$, Inagami $\mathrm{T}$ : Isolation of preferentially expressed genes in the kidneys of hypertensive rats. Hypertension 1991; 17: 161-169.

32. Rapp JP, Dahl LK: Mendelian inheritance of 18 and $11 \beta$-steroid hydroxylase activities in the adrenals of rats genetically susceptible or resistant to hypertension. Endocrinology 1972; 90: 1435-1446.
33. Rapp JP, Dahl LK: Adrenal steroidogenesis in rats bred for susceptibility and resistance to the hypertensive effect of salt. Endocrinology 1971; 88: 52-65.

34. Lindpaintner K, Hilbert P, Ganten D, Nadal-Ginard $\mathrm{B}$, Inagami $\mathrm{T}$, Iwai $\mathrm{N}$ : Molecular genetics of the $\mathrm{S}_{\mathrm{A}^{-}}$ gene: cosegregation with hypertension and mapping to rat chromosome 1 . J Hypertens $1993 ; 11$ : 19-23.

35. Harris EL, Dene $H$, Rapp JP: $S_{A}$ gene and blood pressure cosegregation using Dahl salt-sensitive rats. Am J Hypertens 1993; 6: 330-334.

36. Samani NJ, Lodwick D, Vincent M, et al: A gene differentially expressed in the kidney of the spontaneously hypertensive rat cosegregates with increased blood pressure. J Clin Invest 1993; 92: 10991103.

37. Iwai $\mathrm{N}$, Inagami $\mathrm{T}$ : Identification of a candidate gene responsible for the high blood pressure of spontaneously hypertensive rats. J Hypertens 1992; 10: 1155-1157.

38. Carroll J, Komanicky P, Melby JC: The relationship between plasma 18-hydroxy-11-deoxycorticosterone levels and production of hypertension in the rat. $J$ Steroid Biochem 1981; 14: 989-995.

39. Matsukawa N, Nonaka Y, Higaki J, et al: Dahl's saltresistant normotensive rat has mutations in cytochrome P450(11b), but the salt-sensitive hypertensive rat does not. J Biol Chem 1993; 268: 9117-9121. 